\title{
EFFECT OF LANTANA CAMARA L. COVER ON LOCAL DEPLETION OF TREE POPULATION IN THE VINDHYAN TROPICAL DRY DECIDUOUS FOREST OF INDIA
}

\author{
GYAN P. SHARMA* AND A.S. RAGHUBANSHI \\ Department of Botany, Banaras Hindu University, Varanasi-221005, India. Fax: 91-542- \\ 2368174. Tel : 91-542-2368399. \\ e-mail:gyan_in1@rediffmail.com \\ (Received $31^{\text {th }}$ March 2006; accepted $15^{\text {th }}$ Apr 2007)
}

\begin{abstract}
The dry deciduous forest of northern India is being progressively invaded by an alien invasive woody shrub Lantana camara L. (Lantana). The invasion of lantana threatens the survival of many species. This study examines the demographic instability of tree species at different levels of lantana cover. Based on proportion of seedlings of a species in its total population (seedling+sapling+adult), about $39.5 \%$ and $60 \%$ of total 38 species exhibited local demographic instability at different levels of lantana cover for the first and the second census respectively. This decline in species could be attributed to altered microenvironment (light, $\mathrm{pH}$ and temperature) beneath the lantana bushes. The study concludes that the presence of lantana shrub as dense understorey perturbs the seedling recruitment of native tree species in the forest and this leads to differential depletion of native trees.
\end{abstract}

Key words: declining species population, demographic instability, invasion, lantana cover.

\section{Introduction}

Invasion of native communities by exotic species has been among the most intractable ecological problems of recent years (Sharma et al. 2005a). It is a global scale problem experienced by natural ecosystems and is considered as the second largest threat to global biodiversity (Drake et al., 1989). Despite the recent recognition of the invasion of exotic species as a problem, there are many areas in the world where information on the demographic instability caused by invasion is lacking. In India, especially in the dry deciduous forest region, no information is available on demographic instability caused by lantana (Lantana camara L.). Lantana that ranked top in-terms of highest impacting invasive species (Batianoff \& Butler 2003), and considered one of the worlds 100 worst invasive alien species (GISP 2003), has spread in almost all the areas in the dry deciduous region (Sharma \& Raghubanshi 2006).

Tropical forests occupy 7\% of the Earth's land surface (Wilson 1988) and harbour approximately two third of all biological populations (Hughes et al. 1997). On global basis, $52 \%$ of the total forest are tropical and over $42 \%$ of tropical forest have been classified as dry deciduous (Holdridge 1967). In India, tropical forests account for approximately $86 \%$ of the total forest land (Singh \& Singh 1988) while dry forests are $38.2 \%$ of the total forest cover (MoEF, 1999). These forests are under immense pressure due to various human induced activities. Especially in Vindhyan highlands, quarrying for limestone, establishment of cement factory, thermal power stations and the construction of G B Pant Sagar reservoir have resulted in rapid population increase, causing deforestation and conversion of natural forest ecosystem into marginal croplands (Singh et al. 1991). In fact, during the past 20-years (1981-2001), human population doubled (1463468) in the Sonebhadra district in the Vindhyan region (Rajya 
Niyojan Sansthan, 2000; Anonymous, 2003). Forests around this region are also exposed to illegal sporadic tree felling, wide spread lopping of trees for timber resources and shrubs for fuel wood and leaf for fodder (Singh \& Singh 1989; Jha \& Singh 1990). This rapid modification of the habitat facilitated the invasion of lantana at an accelerated rate (Sharma et al. 2005b), which can subsequently affect species regeneration.

Due to its strong allelopathic properties, Lantana has the potential to interrupt regeneration process of other species by decreasing germination, reducing early growth rates and selectively increasing mortality of other plant species (Sharma et al. 2005ab). These result in a reduction of species diversity and decline of species.

The objective of the present study was to identify the declining species population at low, medium and high lantana cover in the tropical dry deciduous forest of Vindhyan plateau, India. If the ratio of various age groups in a population is known, demography can be used to elucidate the current reproductive status and future trends of that population (Odum 1983; Smith 1996). A large population of young individuals indicates a rapidly expanding population, a more even distribution of age classes a stationary population, and a large population of old individuals a declining population (Odum 1983; Sagar \& Singh 2004). We use the above demographic concept in identifying the depleting species in the areas of lantana invasion.

\section{Material and Methods}

\section{Study area}

The study area lies on the Vindhyan plateau in the Sonebhadra district of Uttar Pradesh $\left(24^{\circ} 13^{\prime}\right.$ to $24^{\circ} 19^{\prime} \mathrm{N} ; 83^{\circ} 59^{\prime}$ to $\left.83^{\circ} 13^{\prime}\right)$ (Fig. 1). The elevation above the mean sea level ranges between 315 and $485 \mathrm{~m}$ (Singh \& Singh 1992). This area has been known as "Sonaghati" (golden valley) due to the richness of its natural resources (Singh et al. 2002).

The climate is tropical with three seasons in a year, i.e. summer (March to mid June), rainy (mid June to September) and winter (October to February). October and March constitute the transition months between the rainy and winter seasons, and between winter and summer seasons, respectively. The average rainfall varies between 850 and $1300 \mathrm{~mm}$. About $85 \%$ of the annual rainfall occurs during the rainy season from the southwest monsoon. The maximum monthly temperature varies from $20^{\circ} \mathrm{C}$ in January to $46^{\circ} \mathrm{C}$ in June, and the mean minimum monthly temperature reaches at $12^{\circ} \mathrm{C}$ in January and at $31^{\circ} \mathrm{C}$ in May.

Red coloured and fine textured sandstone (Dhandraul orthoquartzite) is the most important rock of the area. Sandstone is generally underlain by shale and limestone. The soils derived from these rocks are residual ultisols and are sandy-loam in texture (Raghubanshi 1992). These soils are part of the hyperthermic formation of typical plinthustults with ustorthents according to VII approximation of the USDA soil nomenclature (Singh et al. 2002). The potential natural vegetation of the region is tropical dry deciduous forest, which is locally dominated by species such as Anogeissus latifolia, Boswellia serrata, Buchanania lanzan, Diospyros melanoxylon, Hardwickia binata, Lagerstroemia parviflora, Lannea cormendelica, Madhuca longifolia, Shorea robusta and Terminalia tomentosa. 


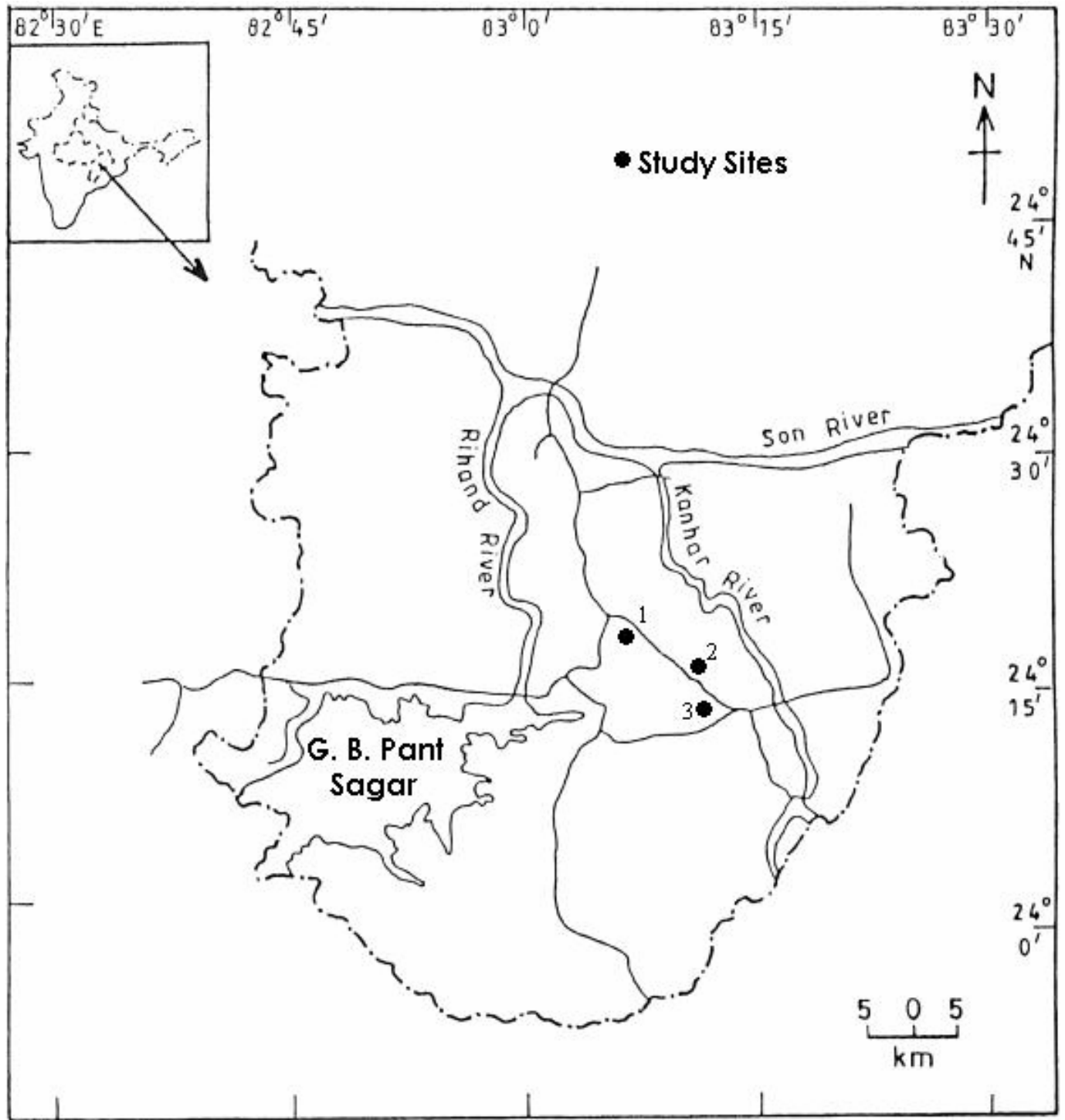

Figure 1. Location of study sites with low, medium and high lantana cover within Vindhyan highlands, India. 1-Hathinala (low); 2- Bhaheradol (medium); 3-Rajkhar (high).

\section{Methods}

Reconnaissance survey of the entire region was made and three sites (Baheradol, Rajkhar and Hathinala) in the region were selected at random. At these sites, which had visually different levels of lantana invasion sampling was done for the year 2002 and 2003 in the month of October. At each site, 30 quadrates each $10 \times 10 \mathrm{~m}$ in size, were sampled randomly for vegetation analysis. A total of 90 quadrates, were sampled for vegetation analysis from the entire study area.

Lantana cover was estimated in each quadrate, using the Domin Krajina scale and was transformed into percentage cover for final analysis (Mueller-Dumbois and Ellenberg 1974). Lantana cover was taken as the percentage of the ground surface 
covered by the shadow of the lantana foliage, estimated as $<1,5,10,30,50,60,75,90$, $>95 \%$, mean values of lantana cover at each site decided its presence in low, medium and high invaded sites. Later, each site was quantified into low (0\%-30\%), medium $(31 \%-60 \%)$ and high (61\%-100\%) invasion sites on the basis of percentage cover of lantana. Data on the canopy cover were collected in each sampling unit in 2002 and 2003. Canopy covers were classified into three categories based on visual estimates of the approximate percentage of overstorey canopy cover- low canopy ; 0-30\% (high light), medium canopy ; 31-60\% (medium light) and high canopy ; 60-100\% (low light). The sites varied in topography; the land was relatively gently sloping at Bhaheradol, undulating at Hathinala site and steep sloping at Rajkhar. These sites also differed in the physico-chemical characteristics of soil and were not related to the lantana cover.

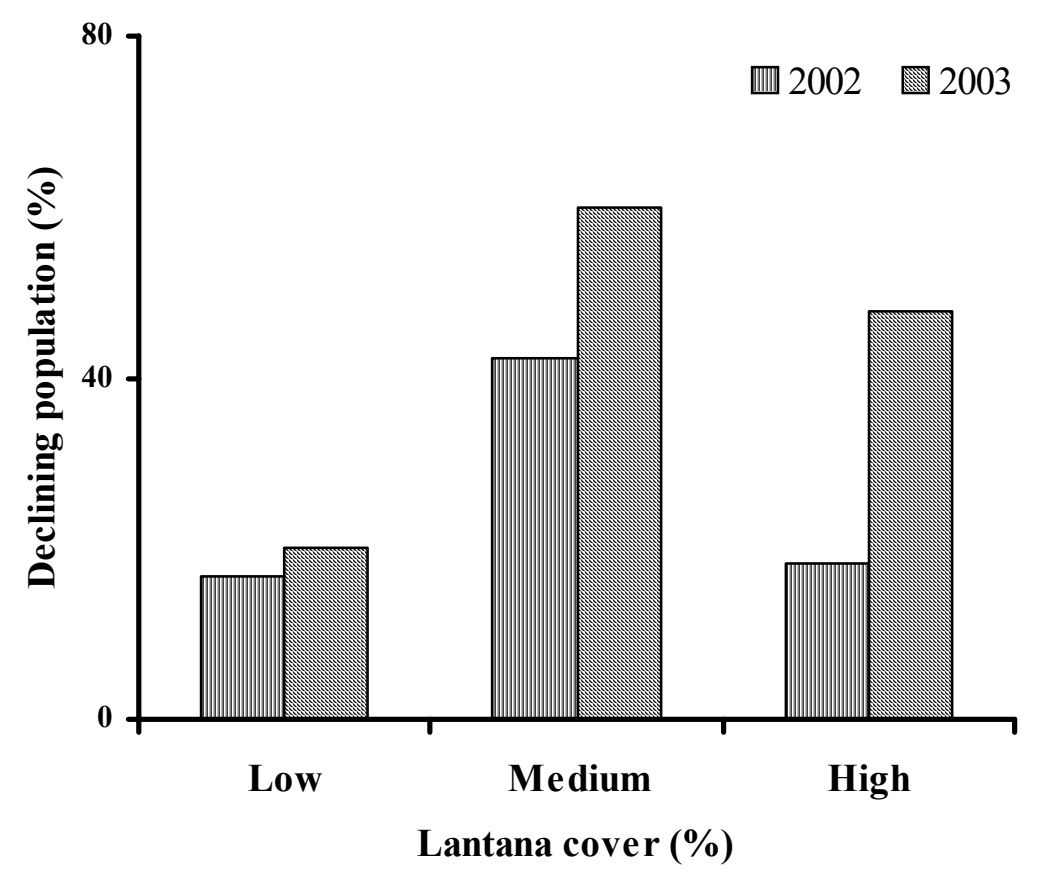

Figure 2. Proportion of declining species at low, medium and high lantana cover within Vindhyan dry deciduous forest of India for two consecutive census (2002 and 2003).

The diameter of each adult individual tree $(\geq 9.6 \mathrm{~cm}$ diameter at breast height, dbh) was measured in each quadrate. In the centre of each $10 \times 10 \mathrm{~m}$ quadrate, a $2 \times 2 \mathrm{~m}$ area was marked for enumeration of saplings (individuals $3.2 \mathrm{~cm}$ to $<9.6 \mathrm{~cm} \mathrm{dbh}$ ) and established seedlings (individuals $<3.2 \mathrm{~cm}$ diameter but $\geq 30 \mathrm{~cm}$ height) (Sagar and Singh 2004). Seedlings shorter than $30 \mathrm{~cm}$ height were considered ephemeral, and the established seedlings category represented 1 to $>3 \mathrm{yr}$ old individuals. Stem diameter of adult and sapling individuals was measured at $1.37 \mathrm{~m}$ from the ground and for seedlings it was measured at $10 \mathrm{~cm}$ above the ground (Sagar and Singh 2004). Thus, all individuals were enumerated and measured by species.

The demographic analysis of species populations on each site was based on the proportion of seedlings of a species in its total population (seedlings + saplings + 
adults). We assumed that for normal replacement, the seedling population should at least comprise more than $50 \%$ of the total population of a species. Based on this assumption we calculated the ratio of seedling population to the total population of a species. The species on each site were classified into four arbitrary categories on the basis of this ratio: $<0.5$ for declining species population (i.e. less than 1 established seedling per mature individual), $0.50-0.66$ for relatively stable population, $0.67-0.99$ for potentially expanding population (i.e. more than 2 seedlings per mature individual) and 1 for newly recruited species from neighbouring areas. A ratio of $<0.25$ (i.e. less than 1 established seedling per 3 mature individuals) was considered to represent severely depleting species population (Sagar \& Singh 2004). Percentage decline at different lantana cover class was obtained by the ratio of number of declining species multiplied by 100 to the total number of species present in different lantana cover classes. However, percentage decline for each year was calculated by the number of declining species in each year multiplied by 100 to the total number of species present in each year.

Relationship between $\mathrm{pH}$, canopy cover, temperature, lantana cover and year of sampling was analyzed by using SPSS software Version 10.0 (SPSS 1997

\section{Result}

In total, 38 species, and 28668 stems were recorded in the cumulative sampled area for both the census with 14851 and 13817 stems for the two consecutive censuses respectively (Table 1). A total of 376 adults, 1500 sapling and 12975 established seedlings were recorded in the first census while 368 adults, 2825 saplings and 10625 established seedlings were recorded in the second census. In total, 7 rare species were recorded, 2 in high and 6 in medium lantana invaded plots. Acacia auriculiformis, Boswellia serrata, Briedelia retusa, Cassia fistula, Elaeodendron glaucum, Eriolena quinquelaris, and Miliusa tomentosa, were recorded as one individual per 0.3 ha sampled area and were distinguished as rare species for both the census. Acacia auriculiformis and Cassia fistula were represented as one individual in entire 0.9 ha sampled area both the census (Table 1).

A total of 15 and 23 species exhibited demographic instability for the two consecutive censuses (Table 2), in other words, local reduction in population size. Of these, 15 species (Acacia auriculiformis, Adina cordifolia, Boswellia serrata, Briedelia retusa, Buchanania lanzan, Cassia fistula, Elaeodendron glaucum, Emblica officinalis, Eriolena quinquelaris, Hardwickia binata, Lannea coromandelica, Miliusa tomentosa, Mitragyna parvifolia, Schleichera oleosa, Schrebera swietenioides) showed relative declining abundances of established seedlings wherever they were present for both the census (Table 2).

Elaeodendron glaucum showed declining population at all the levels of lantana invasion both the census while large number of species with declining population occupied only one or few sites (Table 2). Thus, while 23 species showed declining population at different levels of lantana invasion both the census. The proportion of declining species population was maximum at medium lantana invasion (42 \& $60 \%$ ), than at high lantana invasion $(19 \& 48 \%)$ and minimal at low lantana invasion (16 \& 20 $\%$ ) for both the census respectively. However, the total number of species decreased with increasing lantana cover. 
ANOVA revealed that $\mathrm{pH}$ and temperature significantly varied with the year of sampling, but the canopy cover did not varied significantly with the year (Table 3 ). However, $\mathrm{pH}$, canopy cover and temperature varied significantly with lantana cover (Table 3). Tukey's test revealed that $\mathrm{pH}$, canopy cover and temperature showed significant differences in terms of lantana cover and year of sampling (Table 4).

Table 1. Number of species in different growth categories $(A=$ adult; $S=$ sapling; $E S=$ established seedling) at low, medium and high Lantana camara invasion in a dry tropical forest sites of northern India at temporal scale. Data are number of individuals per 0.3 ha area. Adults were enumerated in 30 quadrats of $10 \times 10 \mathrm{~m}$ size and saplings and established seedlings were enumerated in 30 quadrats of $2 \times 2 \mathrm{~m}$ size at each forest sites. The saplings and established seedling individuals were scaled up in same unit as adult.

\begin{tabular}{|c|c|c|c|c|c|c|c|c|c|c|c|c|c|c|c|c|c|c|}
\hline \multirow[t]{4}{*}{ Species } & \multicolumn{18}{|c|}{ Lantana cover (\%) } \\
\hline & \multicolumn{9}{|c|}{2002} & \multicolumn{9}{|c|}{2003} \\
\hline & \multicolumn{3}{|c|}{ Low } & \multicolumn{3}{|c|}{ Medium } & \multicolumn{3}{|c|}{ High } & \multicolumn{3}{|c|}{ Low } & \multicolumn{3}{|c|}{ Medium } & \multicolumn{3}{|c|}{ High } \\
\hline & A & $\mathrm{S}$ & ES & A & $\mathrm{S}$ & ES & $\mathrm{A}$ & $\mathrm{S}$ & ES & $\mathrm{A}$ & $\mathrm{S}$ & ES & A & $\mathrm{S}$ & ES & A & $\mathrm{S}$ & ES \\
\hline Acacia auriculiformis & 0 & 0 & 0 & 1 & 0 & 0 & 0 & 0 & 0 & 0 & 0 & 0 & 1 & 0 & 0 & 0 & 0 & 0 \\
\hline Acacia catechu Willd. & 12 & 25 & 75 & 19 & 75 & 50 & 0 & 0 & 0 & 12 & 25 & 50 & 20 & 100 & 25 & 0 & 0 & 0 \\
\hline Adina cordifolia Hook. & 0 & 0 & 75 & 0 & 0 & 0 & 4 & 0 & 0 & 0 & 0 & 75 & 0 & 0 & 0 & 4 & 0 & 0 \\
\hline Azadirachta indica A. Juss. & 0 & 0 & 25 & 0 & 0 & 0 & 0 & 0 & 0 & 0 & 0 & 25 & 0 & 0 & 0 & 0 & 0 & 0 \\
\hline $\begin{array}{l}\text { Antidesma ghaesembilla } \\
\text { Gaertn. }\end{array}$ & 0 & 0 & 0 & 0 & 0 & 0 & 0 & 0 & 25 & 0 & 0 & 0 & 0 & 0 & 0 & 0 & 0 & 25 \\
\hline Anogeissus latifolia Wall. & 3 & 25 & 150 & 15 & 25 & 100 & 0 & 50 & 300 & 3 & 25 & 150 & 12 & 25 & 75 & 3 & 100 & 100 \\
\hline Bauhinia racemosa Lam. & 0 & 0 & 150 & 0 & 0 & 0 & 0 & 0 & 0 & 0 & 0 & 150 & 0 & 0 & 0 & 0 & 0 & 0 \\
\hline $\begin{array}{l}\text { Boswellia serrata } \\
\text { Roxb.ex.Colebr. }\end{array}$ & 3 & 0 & 400 & 1 & 0 & 0 & 0 & 0 & 0 & 3 & 0 & 350 & 1 & 0 & 0 & 0 & 0 & 0 \\
\hline $\begin{array}{l}\text { Briedelia retusa Muell - } \\
\text { Arg. }\end{array}$ & 9 & 100 & 800 & 1 & 0 & 0 & 0 & 0 & 25 & 10 & 75 & 750 & 1 & 0 & 0 & 0 & 0 & 25 \\
\hline Buchanania lanzan Spreng. & 10 & 0 & 50 & 5 & 25 & 25 & 3 & 0 & 0 & 10 & 0 & 25 & 4 & 25 & 0 & 3 & 0 & 0 \\
\hline Cassia fistula Linn. & 0 & 0 & 0 & 1 & 0 & 0 & 0 & 0 & 0 & 0 & 0 & 0 & 1 & 0 & 0 & 0 & 0 & 0 \\
\hline Carissa spinarum DC. & 0 & 0 & 0 & 0 & 0 & 0 & 0 & 25 & 75 & 0 & 0 & 0 & 0 & 0 & 0 & 0 & 25 & 75 \\
\hline Casearia elliptica Willet. & 0 & 0 & 0 & 0 & 0 & 0 & 0 & 0 & 150 & 0 & 0 & 0 & 0 & 0 & 0 & 0 & 0 & 150 \\
\hline $\begin{array}{l}\text { Diospyros melanoxylon } \\
\text { Roxb. }\end{array}$ & 6 & 25 & 625 & 9 & 50 & $\begin{array}{c}110 \\
0\end{array}$ & 2 & 100 & 950 & 7 & 0 & 550 & 10 & 25 & 1050 & 3 & 225 & 700 \\
\hline Elaeodendron glaucum Pers. & 2 & 0 & 0 & 2 & 0 & 0 & 1 & 0 & 0 & 2 & 0 & 0 & 2 & 0 & 0 & 1 & 0 & 0 \\
\hline Emblica officinalis Gaerth. & 6 & 25 & 50 & 1 & 0 & 0 & 0 & 0 & 25 & 6 & 25 & 50 & 1 & 0 & 0 & 0 & 0 & 0 \\
\hline
\end{tabular}

\begin{tabular}{|l|c|c|c|c|c|c|c|c|c|c|c|c|c|c|c|c|c|c|}
\hline $\begin{array}{l}\text { Eriolaena quinquelocularis } \\
\text { Wight. }\end{array}$ & 0 & 0 & 125 & 1 & 0 & 0 & & & & 0 & 0 & 125 & 1 & 0 & 0 & & 0 & 0 \\
\hline Flacourtia indica Murr. & 0 & 0 & 250 & 2 & 0 & 200 & 0 & 0 & 25 & 0 & 0 & 250 & 2 & 0 & 150 & 0 & 0 & 100 \\
\hline Gardenia latifolia Ait. & 1 & 25 & 125 & 1 & 0 & 25 & 0 & 0 & 0 & 1 & 25 & 125 & 1 & 25 & 25 & 0 & 0 & 0 \\
\hline Grewia serrulata DC. & 0 & 0 & 275 & 0 & 0 & 0 & 0 & 0 & 0 & 0 & 75 & 175 & 0 & 0 & 0 & 0 & 0 & 0 \\
\hline Hardwickia binnata Roxb. & 5 & 0 & 0 & 3 & 25 & 0 & 0 & 0 & 0 & 5 & 0 & 0 & 3 & 25 & 0 & 0 & 0 & 0 \\
\hline
\end{tabular}




\begin{tabular}{|l|c|c|c|c|c|c|c|c|c|c|c|c|c|c|c|c|c|c|}
\hline $\begin{array}{l}\text { Holarrhena antidysenterica } \\
\text { DC. }\end{array}$ & 0 & 0 & 0 & 105 & 0 & 150 & 0 & 0 & 200 & 0 & 150 & 875 & 1 & 0 & 150 & 0 & 25 & 75 \\
\hline $\begin{array}{l}\text { Hymenodictyon excelsum } \\
\text { Wall. }\end{array}$ & 0 & 0 & 100 & 0 & 0 & 25 & 0 & 0 & 0 & 0 & 0 & 100 & 0 & 0 & 0 & 0 & 0 & 0 \\
\hline $\begin{array}{l}\text { Lagerstroemia parviflora } \\
\text { Roxb. }\end{array}$ & 4 & 0 & 50 & 14 & 25 & 100 & 1 & 50 & 75 & 4 & 0 & 50 & 14 & 25 & 100 & 2 & 50 & 25 \\
\hline $\begin{array}{l}\text { Lannea coromandelica } \\
\text { Merr. }\end{array}$ & 17 & 25 & 0 & 3 & 50 & 25 & 17 & 25 & 75 & 17 & 25 & 0 & 3 & 50 & 25 & 17 & 25 & 25 \\
\hline Madhuca longifolia MacBr. & 0 & 0 & 0 & 0 & 0 & 0 & 12 & 0 & 25 & 0 & 0 & 0 & 0 & 0 & 0 & 10 & 0 & 25 \\
\hline Miliusa tomentosa Sincl. & 3 & 25 & 175 & 3 & 100 & 450 & 1 & 0 & 0 & 3 & 50 & 125 & 3 & 200 & 300 & 1 & 0 & 0 \\
\hline
\end{tabular}

\begin{tabular}{|c|c|c|c|c|c|c|c|c|c|c|c|c|c|c|c|c|c|c|}
\hline Mitragyna Parvifolia Korth. & 0 & 0 & 25 & 0 & 0 & 0 & 0 & 0 & 0 & 0 & 25 & 0 & 0 & 0 & 0 & 0 & 0 & 0 \\
\hline $\begin{array}{l}\text { Pterocarpus marsupium } \\
\text { Roxb. }\end{array}$ & 0 & 0 & 25 & 0 & 0 & 0 & 0 & 0 & 0 & 0 & 0 & 25 & 0 & 0 & 0 & 0 & 0 & 0 \\
\hline Schleichera oleosa Oken. & 0 & 0 & 0 & 0 & 0 & 0 & 0 & 0 & 25 & 0 & 0 & 0 & 0 & 0 & 0 & 0 & 25 & 0 \\
\hline $\begin{array}{l}\text { Schrebera swietenioides } \\
\text { Roxb. }\end{array}$ & 9 & 0 & 0 & 0 & 0 & 25 & 0 & 0 & 0 & 9 & 0 & 0 & 0 & 25 & 0 & 0 & 0 & 50 \\
\hline $\begin{array}{l}\text { Semecarpus anacardium } \\
\text { Linn.f. }\end{array}$ & 2 & 25 & 300 & 0 & 25 & 50 & 0 & 0 & 25 & 2 & 75 & 250 & 0 & 50 & 25 & 0 & 0 & 25 \\
\hline Shorea robusta Gaertn. & 52 & 75 & 650 & 39 & 50 & 450 & 31 & 75 & 675 & 48 & 175 & 550 & 34 & 175 & 425 & 28 & 325 & 300 \\
\hline Soymida febrifuga A. Juss & 4 & 25 & 25 & 1 & 0 & 75 & 0 & 0 & 0 & 4 & 25 & 25 & 1 & 50 & 25 & 0 & 0 & 0 \\
\hline Sterculia urens Roxb. & 2 & 50 & 100 & 0 & 0 & 50 & 0 & 0 & 0 & 2 & 50 & 100 & 0 & 25 & 50 & 0 & 0 & 0 \\
\hline $\begin{array}{l}\text { Terminalia tomentosa } \\
\text { Wight. }\end{array}$ & 8 & 25 & $\begin{array}{c}107 \\
5\end{array}$ & 12 & 175 & 275 & 11 & 75 & 200 & 7 & 25 & 1025 & 12 & 200 & 250 & 12 & 125 & 75 \\
\hline $\begin{array}{l}\text { Zizyphus nummularia Wight } \\
\text { \& Arn. }\end{array}$ & 0 & 0 & 75 & 0 & 0 & 50 & 0 & 0 & 0 & 0 & 25 & 75 & 0 & 0 & 50 & 0 & 0 & 0 \\
\hline Sikti (unidentified) & 0 & 0 & 0 & 0 & 0 & 0 & 0 & 0 & 50 & 0 & 0 & 0 & 0 & 0 & 0 & 0 & 0 & 75 \\
\hline
\end{tabular}

Table 2: Species showing local population depletion (*) based on the $<0.50$ ratio of seedling population to total species population at low, medium and high Lantana camara invasion at temporal scale. Zeroes indicate complete absence of established seedlings. Values are ratios of established seedlings to total population (- = species not present).

\begin{tabular}{|c|c|c|c|c|c|c|c|c|}
\hline \multirow[t]{3}{*}{ Species } & \multirow[t]{3}{*}{ Family } & \multirow[t]{3}{*}{ Common names } & \multicolumn{6}{|c|}{ Lantana cover } \\
\hline & & & \multicolumn{3}{|c|}{2002} & \multicolumn{3}{|c|}{2003} \\
\hline & & & Low & Medium & High & Low & Medium & High \\
\hline Acacia auriculiformis & Mimosiaceae & Australian babool & - & $0.00^{*}$ & - & - & $0.00 *$ & - \\
\hline Acacia catechu Willd. & Mimosiaceae & Khair & 0.67 & $0.35^{*}$ & - & 0.57 & $0.17^{*}$ & - \\
\hline Adina cordifolia Hook. & Rubiaceae & Haldu/Karam & 1.00 & - & $0.00 *$ & 1.00 & - & $0.00 *$ \\
\hline Azadirachta indica A. Juss. & Miliaceae & Neem & - & - & 1.00 & 1.00 & - & - \\
\hline Antidesma ghaesembilla Gaertn. & Euphorbiaceae & Sahrauta & 1.00 & - & - & - & - & 1.00 \\
\hline Anogeissus latifolia Wall. & Combrataceae & Dhaura/Dhau & 0.84 & 0.71 & 0.86 & 0.84 & 0.67 & $0.49 *$ \\
\hline Bauhinia racemosa Lam. & Caesalpiniaceae & Katahul & 1.00 & - & - & 1.00 & - & - \\
\hline Boswellia serrata Roxb.ex.Colebr. & Burseracaeae & Salai & 0.99 & $0.00^{*}$ & - & 0.99 & $0.00^{*}$ & - \\
\hline Briedelia retusa Muell - Arg. & Euphorbiacae & Khaja & 0.88 & $0.00^{*}$ & 1.00 & 0.90 & $0.00^{*}$ & 1.00 \\
\hline Buchanania lanzan Spreng. & Anacardiaceae & Piyar & 0.83 & $0.45^{*}$ & $0.00 *$ & 0.71 & $0.00 *$ & $0.00 *$ \\
\hline Carissa spinarum DC. & Apocynaceae & Karaunda & - & - & 0.75 & - & - & 0.75 \\
\hline Casearia elliptica Willet. & Flacourtiaceae & Bheri & - & - & 1.00 & - & - & 1.00 \\
\hline Cassia fistula Linn. & Caesalpiniaceae & Amaltas & - & $0.00^{*}$ & - & - & $0.00^{*}$ & - \\
\hline Diospyros melanoxylon Roxb. & Ebenaceae & Tendu & 0.95 & 0.95 & 0.90 & 0.99 & 0.97 & 0.75 \\
\hline Elaeodendron glaucum Pers. & Celastraceae & Mamar & $0.00^{*}$ & $0.00^{*}$ & $0.00 *$ & $0.00 *$ & $0.00^{*}$ & $0.00 *$ \\
\hline
\end{tabular}




\begin{tabular}{|c|c|c|c|c|c|c|c|c|}
\hline Emblica officinalis Gaerth. & Euphorbiacae & Aonla & 0.62 & $0.00^{*}$ & 1.00 & 0.62 & $0.00 *$ & - \\
\hline Eriolaena quinquelocularis Wight. & Sterculiaceae & Dheriya & 1.00 & $0.00 *$ & - & 1.00 & $0.00 *$ & - \\
\hline Flacourtia indica Murr. & Flacourtiaceae & Kantaila & 1.00 & 0.99 & 1.00 & 1.00 & 0.99 & 1.00 \\
\hline Gardenia latifolia Ait. & Rubiaceae & Papar & 0.83 & 0.96 & - & 0.83 & $0.49 *$ & - \\
\hline Grewia serrulata DC. & Tiliaceae & Bichhula & 1.00 & - & - & 0.70 & - & - \\
\hline Hardwickia binnata Roxb. & Caesalpiniaceae & Parsiddha & $0.00 *$ & $0.00 *$ & - & $0.00 *$ & $0.00 *$ & - \\
\hline Holarrhena antidysenterica DC. & Apocynaceae & Khirana/Koraya & 1.00 & 0.99 & 1.00 & 0.85 & 0.99 & 0.75 \\
\hline Hymenodictyon excelsum Wall. & Rubiaceae & Bhurkul & 1.00 & 1.00 & - & 1.00 & - & - \\
\hline Lagerstroemia parviflora Roxb. & Lythraceae & Siddha & 0.93 & 0.72 & 0.60 & 0.93 & 0.72 & $0.32 *$ \\
\hline Lannea coromandelica Merr. & Anacardiaceae & Gigan & $0.00 *$ & $0.32 *$ & 0.64 & $0.00 *$ & $0.32 *$ & $0.37 *$ \\
\hline Madhuca longifolia MacBr. & Sapotaceae & Mahua & - & - & 0.68 & & & 0.71 \\
\hline Miliusa tomentosa Sincl. & Anonaceae & Kari & 0.86 & 0.81 & $0.00 *$ & 0.70 & 0.60 & $0.00 *$ \\
\hline Mitragyna Parvifolia Korth. & Rubiaceae & Gurahi & 1.00 & - & - & $0.00^{*}$ & - & - \\
\hline Pterocarpus marsupium Roxb. & Fabaceae & Biya/Vijayasal & 1.00 & - & - & 1.00 & - & - \\
\hline Schleichera oleosa Oken. & Sapindaceae & Kusum & - & - & 1.00 & - & - & $0.00 *$ \\
\hline Schrebera swietenioides Roxb. & Oleaceae & Ghantha & $0.00 *$ & 1.00 & 1.00 & $0.00 *$ & $0.00^{*}$ & 1.00 \\
\hline Semecarpus anacardium Linn.f. & Anacardiaceae & Bhela & 0.92 & 1.00 & 1.00 & 0.76 & $0.33 *$ & 1.00 \\
\hline Shorea robusta Gaertn. & Dipterocarpaceae & Sal/Shakhu & 0.84 & 0.67 & 0.86 & 0.71 & 0.67 & $0.46^{*}$ \\
\hline Soymida febrifuga A. Juss & Miliaceae & Rohina & $0.46^{*}$ & 0.83 & - & $0.46^{*}$ & $0.33^{*}$ & - \\
\hline Sterculia urens Roxb. & Sterculiaceae & Kuruli & 0.66 & 0.99 & - & 0.66 & 0.67 & - \\
\hline Terminalia tomentosa Wight. & Combrataceae & Asan/Saja & 0.97 & 0.60 & 0.70 & 0.97 & 0.54 & $0.35^{*}$ \\
\hline Zizyphus nummularia Wight \& Arn. & Rhamnaceae & Ber/Beri & 1.00 & 1.00 & - & 0.75 & 1.00 & - \\
\hline Sikti (unidentified) & & & - & - & 1.00 & - & - & 1.00 \\
\hline
\end{tabular}

Table 3. Summary of ANOVA for $\mathrm{pH}$, Canopy cover, temperature in response to year and lantana cover.

\begin{tabular}{|l|l|l|c|c|}
\hline Source of variation & Dependent variable & df & F & P \\
\hline \multirow{4}{*}{ Year } & $\mathrm{pH}$ & 1 & 64.04 & 0.000 \\
\cline { 2 - 5 } & Canopy cover $(\%)$ & 1 & 1.06 & 0.312 \\
\cline { 2 - 5 } & Temperature $\left({ }^{\circ} \mathrm{C}\right)$ & 1 & 10.46 & 0.004 \\
\hline \multirow{3}{*}{ Lantana cover $(\%)$} & $\mathrm{pH}$ & 2 & 24.06 & 0.000 \\
\cline { 2 - 5 } & Canopy cover $(\%)$ & 2 & 180.413 & 0.000 \\
\cline { 2 - 5 } & Temperature $\left({ }^{\circ} \mathrm{C}\right)$ & 2 & 47.26 & 0.000 \\
\hline \multirow{3}{*}{ Year $\times$ Lantana cover $(\%)$} & $\mathrm{pH}$ & 2 & 0.67 & 0.51 \\
\cline { 2 - 5 } & Canopy cover $(\%)$ & 2 & 0.54 & 0.58 \\
\cline { 2 - 5 } & Temperature $\left({ }^{\circ} \mathrm{C}\right)$ & 2 & 0.68 & 0.51 \\
\hline Error & & 24 & & \\
\hline Total & & 30 & & \\
\hline
\end{tabular}


Table 4. Data on pH, temperature and canopy cover at different lantana cover for the two consecutive years.

\begin{tabular}{|c|c|c|}
\hline \multirow[t]{2}{*}{ Lantana cover (\%) } & \multicolumn{2}{|c|}{ Year } \\
\hline & 2002 & 2003 \\
\hline & \multicolumn{2}{|c|}{ pH } \\
\hline Low & $6.65 \pm 0.02^{\mathrm{a}}$ & $6.86 \pm 0.02^{d}$ \\
\hline Medium & $6.52 \pm 0.04^{\mathrm{b}}$ & $6.72 \pm 0.05^{b}$ \\
\hline \multirow[t]{2}{*}{ High } & $6.38 \pm 0.03^{\mathrm{c}}$ & $6.65 \pm 0.03^{\mathrm{e}}$ \\
\hline & \multicolumn{2}{|c|}{ Temperature $\left({ }^{\circ} \mathrm{C}\right)$} \\
\hline Low & $33.22 \pm 0.45^{\mathrm{a}}$ & $32.61 \pm 0.36^{\circ}$ \\
\hline Medium & $30.96 \pm 0.35^{b}$ & $29.73 \pm 0.65^{b}$ \\
\hline \multirow[t]{2}{*}{ High } & $29.53 \pm 0.34^{\mathrm{c}}$ & $27.92 \pm 0.38^{\circ}$ \\
\hline & \multicolumn{2}{|c|}{ Canopy cover $(\%)$} \\
\hline Low & $71 \pm 1.87^{\mathrm{a}}$ & $72 \pm 2.55^{\mathrm{a}}$ \\
\hline Medium & $50 \pm 2.24^{\mathrm{b}}$ & $46 \pm 1.87^{\mathrm{d}}$ \\
\hline High & $21 \pm 3.67^{\mathrm{c}}$ & $17 \pm 3.74^{\mathrm{e}}$ \\
\hline
\end{tabular}

\section{Discussion}

Sagar and Singh (2004) reported 12 species (viz. Boswellis serrata, Carrissa spinarum, Cassia fistula, Cassia siamea, Dalbergia sissoo, Ficus benghalensis, Holoptelia integrifolia, Madhuca latifolia, Syzygium heyneanum, Terminalia tomentosa, 2 unidentified species- Papra and Rij) showing a reduction in population size at varied disturbance regimes. Study conducted at various levels of lantana cover revealed that the species with declining population differed, except for Boswellis serrata and Cassia fistula which showed declining populations at various disturbance regimes (Sagar \& Singh 2004). Thirteen more species, other than those with declining population due to disturbance, showed a decline at different levels of lantana invasion for both the census. The number of declining species increased when both the phenomena (viz. disturbance and lantana invasion) were taken into consideration. Thus, we may interpret that disturbance and invasion synergistically affect the tree regeneration process in the Vindhyans. Although, Sharma et al. (2005c) emphasized that while studying invasiveness, all the available tenets must be taken into consideration to explain invasions in totality.

Species exhibited as a single individual at different lantana cover included Acacia auriculiformis, Boswellia serrata, Briedelia retusa, Cassia fistula, Emblica officinalis and Eriolena quinquelaris at medium lantana invasion and Elaeodendron glaucum and 
Miliusa tomentosa at high lantana invasion (Table 1). It is obvious that the species with only one individual would be highly vulnerable, since a local population composed of only a few individuals can undergo catastrophic decline due to environmental change, genetic problem or simple random events when isolated in a limited geographic range (Cunningham \& Saigo 1999). According to Barbault \& Sastrapradja (1995), species with small population size are highly vulnerable and severe the environmental change the higher the rate of local loss of species population. A minimum size of population is required for long-term viability of rare and endangered species (Cunningham \& Saigo 1999).

The forest supplies approximately $90 \%$ of the fuel wood and fodder needs of the local population. Exploitation of single species can cause the entire structure of the plant community to change (Spurr \& Barnes 1980). Further, fuel wood collection can be a major contributor to forest degradation. These result in canopy openings, which permit lantana to flourish well and affect the tree regeneration process due to competition. Under such conditions, shading effect and allelopathic activity of lantana cause seedlings of tree species have stunted or no growth. When canopy trees are removed patches of increased light intensity (Rejmanek 1989) and nutrient resources (Davis et al. 2000) are created. Lantana is faster growing than native tree species and it captures the resources efficiently, creating substantial biomass of lantana in the intercanopy and at the edges of the forest (personal observation). Breshears (2006) also emphasized that canopy opening (in terms of cover) affects ecosystem functioning between canopy and inter-canopy patches in terms of light irradiance. Rodger \& Twine (2002) advocated that lantana invasion could be considered as a form of bush encroachment. The dense growth of lantana in the form of understorey mat prevents the light to reach the ground, resulting in marked heterogeneity in terms of irradiance and temperature. Light has long been recognized as an important plant resource (Maximov 1929; Blankenship 2002) that may interact with other plant resources to affect plant performance (Cole 2003). Below certain thresholds, however, light limitation alone can prevent seedling survival regardless of other resource levels (Tilman 1982). It is likely that seedlings of tree species are influenced by the amount of light that reaches the forest floor, and this may be probably one of the mechanisms responsible for the decline of seedlings. Sharma and Raghubanshi 2006 advocated that the growth architecture pattern of lantana is such that it prevents the light penetration to the forest floor, leading to the decline of tree seedlings.

Almost all the species with declining population (Acacia auriculiformis, Adina cordifolia, Boswellia serrata, Briedelia retusa, Buchanania lanzan, Cassia fistula, Elaeodendron glaucum, Emblica officinalis, Eriolena quinquelaris, Hardwickia binata, Miliusa tomentosa, Schrebera swietenioides) require high to moderate light for proper growth (Troup 1921). Lantana canopy certainly lowers sunlight to reach the ground level, which may affect the soil temperature. The dry forest tree species are fairly tolerant to moderately high temperatures at all the life cycle stages (Khurana and Singh 2001a). As increase in temperature triggers germination by changing the internal enzymatic kinetics and thus the biochemistry of seed cells (Khurana and Singh 2001b, Vazquez and Orozeo 1982). However, the decrease in temperature due to lantana canopy might perturb seedling recruitment. This may possibly be one of the reasons that inhibits seedling establishment of the tree species. Further, the change in $\mathrm{pH}$ that may be attributed to the allelopathic potential of lantana and this could have important implication for the seedling establishment. 
Lantana bushes are fire prone and can burn readily altering the fire regime to favour its persistence (Hiremath and Sundaram 2005). These lantana bushes when burned create ample heat, causing seed and seedling mortality in the area (Moore \& Wein 1977). Thus, interaction of these factors with biotic pressure might inhibit both seed germination as well as seedling establishment, which may result in population loss.

There are several ways in which lantana could suppress tree recruitment selectively. As lantana forms dense bushes and when such bushes grow nearby each other they form an understorey mat like structure (personal observation). And such mat formation prevents the seeds of tree species to reach the ground; these seeds get entrapped in the lantana mat. For example, the fruit of Schrebera swietenioides is 2-3 inch long (Brandis 1978), which can be easily trapped in lantana mat. Although when few seeds reach the ground there is scarcity of light. At ground level there is accumulation of lantana litter and other broken dry debris of lantana, which could lead to allelopathic suppression for germination of seeds of tree species (Gentle \& Duggin 1997).

\section{Conclusion}

This study suggests that not only many species of the tropical dry deciduous forest have small local population, but also several of them exhibit declining or even severely depleting population at different levels of lantana invasion. Such invasive species cover may create demographic instability among the tree species and reduce tree diversity and can even change the structure of the forest in the near future. As seedlings of most of the tree species of tropical dry deciduous forest are adapted to grow in relatively open conditions, because of the poor canopy cover and deciduous nature. The presence of lantana shrub as dense understorey perturbs the seedling recruitment of native tree species in the forest and this lead to differential depletion of native trees. This calls for immediate conservation activity. Further this study gives an implication and need for indepth microcosm study in relation to lantana specific performance of declining species in the tropical dry deciduous forest.

Acknowledgements. Funding support from Council of Scientific and Industrial Research, New Delhi, in form of a Junior Research Fellowship to Gyan P Sharma is gratefully acknowledged. The authors thank Prof. Jamuna Sharan Singh, Department of Botany, Banaras Hindu University Varanasi, India for his thoughtful comments and critical suggestions.

\section{REFERENCES}

[1] Anonymous. 2003. District of India. Available from URL: www.statoids.com/yin.html Accessed on 5 June 2005.

[2] Barbault R. and Sastrapradja S. 1995. Generation, maintenance and loss of biodiversity. In: Global Biodiversity Assessment (ed by Heywood V. H. and Watson R. T.) Cambridge University Press pp. 194-274.

[3] Batianoff G. N. and Butler D.W. 2003. Impact assessment and analysis of sixty-six priority invasive weeds in southeast Queensland. Pl. Prot. Quart. 18, 11-17.

[4] Blankenship R.E. 2002. Molecular Mechanisms of Photosynthesis. Blackwell Science, Oxford. 
[5] Brandis D. 1978. Indian Trees. Jayyed press, Ballimaran, New Delhi, India, 767pp.

[6] Breshears D.D. 2006. The grassland-forest continuum: trends in ecosystem properties for woody plant mosaics? Front. Ecol. Environ. 4, 96-104.

[7] Cole P.G. 2003. Environmental constraints on the distribution of the non-native invasive grass, Microstegium vimineum. PhD Thesis, Department of Ecology and Evolutionary Biology, University of Tennessee, Knoxville, TN.

[8] Cunningham W. P. and Saigo B. W. 1999 Environmental Sciences: A Global Concern. The McGraw-Hill Companies, Inc.

[9] Davis M. A., Grime J. P. and Thompson K. 2000. Fluctuating resources in plant communities: a general theory of invasibility. J. Ecol. 88, 528-534.

[10] Drake J. A., di Castri F., Grooves R. H., Druger F. J., Mooney H. A., Rejmanek M. and Williamson M. (eds) 1989. Biological Invasion: a Global Perspective. SCOPE 37. John Wiley \& Sons Ltd., Chichester, U.K., 525 pp.

[11] Gentle C. B. and Duggin J. A. 1997. Allelopathy as a competitive strategy in persistent thickets of Lantana camara L. in three Australian forest communities. Pl. Ecol. 132, 8595.

[12] GISP. 2003. The IAS problem. The Global Invasive Species Programme, Available from URL: http://www.gisp.org/about/IAS.asp Asscessed 12 February 2005.

[13] Hiremath A.J. and Sundaram B. 2005. The fire-Lantana cycle hypothesis in Indian forests. Conserv. Soc. 3, 26-42

[14] Holdridge L. R. 1967. Life Zone Ecology. San Jose, Costa Rica: Tropical Science Center.

[15] Hughes J. B., Daily G. C. and Ehrlich P. R. 1997. Population diversity: its extent and extinction. Science 278, 689-692.

[16] Jha C. S. and Singh J. S. 1990. Composition and dynamics of dry tropical forests in relation to soil texture. J. Veg. Sci. 1, 609-614.

[17] Khurana, E and Singh, J. S. 2001a. Ecology of seed and seedling growth for conservation and restoration of tropical dry forest: a review. Environ. Conserv. 28, 39-52.

[18] Khurana, E and Singh, J. S. 2001b. Ecology of tree seed and seedlings: implication for tropical forest conservation and restoration. Curr. Sci. 80, 748-757.

[19] Maximov N.A. 1929. The Plant in Relation to Water. George Allen \& Unwin, London.

[20] MoEF. 1999. National Policy and Macrolevel Action Strategy on Biodiversity. Ministry of Environment \& Forests, Government of India, New Delhi.

[21] Moore J. M. and Wein R. W. 1977. Viable seed population by soil depth and potential site recolonization after disturbance. Can. J. Bot. 55, 2408-2412.

[22] Mueller-Dumbois D. and Ellenberg H. 1974. Aims and methods of vegetation ecology. John Wiley \& Sons, New York.

[23] Odum E. P. 1983. Basic Ecology. Saunders College Publishing, Philadelphia New York.

[24] Raghubanshi A. S. 1992. Effect of topography on selected soil properties and nitrogen mineralization in a dry tropical forest. Soil Biol. Biochem. 24, 145-150.

[25] Rajya Niyojan Sansthan. 2000. Sankhyikiya Patrika: Janpad Sonbhadra. Karyalaya Arth aunm Sankhya Prabhag, Rajya Niyojan Sansthan, Uttar Pradesh, India.

[26] Rejmanek M. 1989. Invasibility of plant communities. In: Biological Invasion: a Global Perspective. SCOPE 37 (ed. by Drake J. A., di Castri F., Grooves R. H., Druger F. J., Mooney H. A., Rejmanek M. and Williamson M.) pp 281-300, John Wiley \& Sons Ltd., Chichester, U.K.

[27] Rodger J. G. and Twine W. C. 2002. Tree canopies facilitate invasion of communal savanna rangelands Lantana camara. Afr. J. Range Forage Sci. 19, 131-135.

[28] Sagar R. and Singh J. S. 2004. Local plant species depletion in a tropical dry deciduous forest of northern India. Environ. Conserv. 31, 55-62.

[29] Sharma G.P. and Raghubanshi A.S. (2006) Tree population structure, regeneration and expected future composition at different levels of Lantana camara L. invasion in the Vindhyan tropical dry deciduous forest of India. Lyonia. 11, 25-37 
[30] Sharma G.P., Singh J.S. and Raghubanshi A.S. (2005a) Plant invasions: emerging trends and future implications. Curr. Sci. 88, 726-734

[31] Sharma G.P., Raghubanshi A.S. and Singh J.S. (2005b) Lantana invasion: an overview. Weed Biol. Manag. 5, 157-167

[32] Sharma G.P., Raghubanshi A.S. and Singh J.S. (2005c) Plant invasion hypothesis in complimentarity. Science.

[33] Available from http://www.sciencemag.org/cgi/eletters/310/5746/243. Accessed 8 December 2005

[35] Singh A. K., Raghubanshi A. S. and Singh J. S. 2002. Medical ethnobotany of the tribals of Sonaghati of Sonebhadra district, Uttar pradesh, India. J. Ethnopharmacology 81, 3141.

[36] Singh J. S. and Singh V. K. 1992. Phenology of seasonally dry tropical forest. Curr. Sci. 63, 684-688.

[37] Singh J. S., Singh K. P. and Agrawal M. 1991. Environmental degradation of the ObraRenukoot-Singrauli area, India and its impact on natural and derived ecosystems. The Environmentalist 11, 171-180.

[38] Singh V. P. and Singh J. S. 1989. Man and forest: a case study from the dry tropics of India. Environ. Conserv. 16, 129-136.

[39] Singh K. P. and Singh J. S. 1988. Certain structural and functional aspects of dry tropical forests and savanna. Int. J. Ecol. Environ. Sci. 14, 31-45.

[40] Smith R. L. 1996. Ecology and Field Biology. Harper Collins college publishers, Inc. New York.

[41] SPSS 1997. SPSS Base7.5 Application Guide. SPSS, Chicago, 389pp

[42] Spurr S. H. and Barnes B. V. 1980. Forest Ecology. New York, John Wiley.

[43] Tilman D. 1982. Resource Competition and Community Structure. Princeton University Press, NJ.

[44] Troup R. S. 1921. The Silviculture of Indian Trees vol. I and II. Oxford: at the Clarendon Press.

[45] Vazquez-Yanes, C. and Orozeo-Segovia, A. 1982. Seed germination of a tropical rain forest pioneer tree Heliocarpus donnell-smithii in response to diurnal fluctuation of temperature. Physiol. Planta. 56, 295-298.

[46] Wilson E. O. 1988. The current state of biological diversity. In: Biodiversity (ed. by Wilson E. O. and. Peter F. M.) pp3-18, Washington, D.C: National Academy Press. 